\title{
Correlates of short- and long-term case fatality within an incident stroke population in Tanzania
}

\author{
R W Walker, A Jusabani, E Aris, W K Gray, F Mugusi, M Swai, K G Alberti, N Unwin \\ North Tyneside General Hospital, Tyne and Wear, UK \\ $\mathrm{R}$ W Walker, MD \\ W K Gray, PhD \\ Institute of Health and Society, University of Newcastle-upon-Tyne, UK \\ R W Walker, MD \\ N Unwin, DM
}

Kilimanjaro Christian Medical Centre, Moshi, Tanzania

A Jusabani, MD

M Swai, MD

Department of Neurology, Muhimbili University Hospital, Dar-es-Salaam, Tanzania

E Aris, MD

F Mugusi, MD

Department of Endocrinology and Metabolism, St Mary's Hospital Campus, Imperial College, London, UK K G Alberti, FRCP

Faculty of Medical Sciences, Cave Hill Campus, University of the West Indies, Barbados N Unwin, DM

Corresponding author: R W Walker (richard.walker@nhct.nhs.uk)

\begin{abstract}
Background. This study aimed to identify correlates of case fatality within an incident stroke population in rural Tanzania.
Methods. Stroke patients, identified by the Tanzanian Stroke Incidence Project, underwent a full examination and assessment around the time of incident stroke. Records were made of demographic data, blood pressure, pulse rate and rhythm, physical function (Barthel index), neurological status (communication, swallowing, vision, muscle activity, sensation), echocardiogram, chest X-ray and computed tomography (CT) head scan. Cases were followed up over the next 3 - 6 years.

Results. In 130 incident cases included in this study, speech, language and swallowing problems, reduced muscle power, and reduced physical function were all significantly correlated with case fatality at 28 days and 3 years. Age was significantly correlated with case fatality at 3 years, but not at 28 days post-stroke. Smoking history was the only significant correlate of case fatality at 28 days that pre-dated the incident stroke. All other significant correlates were measures of neurological recovery from stroke.

Conclusions. This is the first published study of the correlates of post-stroke case fatality in sub-Saharan Africa (SSA) from an incident stroke population. Case fatality was correlated with the various motor impairments resulting from the incident stroke. Improving poststroke care may help to reduce stroke case fatality in SSA.

S Afr Med J 2013;103(2):107-112. DOI:10.7196/SAMJ.5793
\end{abstract}

Stroke remains the third most common cause of death in high-income countries. ${ }^{1,2}$ Nevertheless, stroke incidence and case fatality rates appear to be falling in these regions of the world, and this is likely to be due to stroke prevention initiatives and improved post-stroke care. ${ }^{3-6}$ In low- and middle-income regions, the situation is more complex. With limited provision of primary healthcare in many regions, poststroke mortality rates remain high, and demographic changes mean that as populations age, rates of stroke-attributable mortality are likely to continue to rise. ${ }^{4-6}$

We have recently reported post-stroke case fatality rates from an incident population in rural Tanzania. ${ }^{7}$ Fatality rates at 28 days and at 3 years were higher than are seen in high-income countries, and it was suggested that mortality rates may be reduced by improved post-stroke care. Identifying correlates of post-stroke case fatality may help healthcare providers to target resources at those areas where they may be most effective.
A number of studies from Europe and North American have tried to identify predictors of post-stroke case fatality. The American Cardiovascular Health Study found that, in 455 cases, post-stroke mortality over 7 years was predicted by greater age, haemorrhagic or cardioembolic stroke and pre-stroke poor performance in a timed walking test. ${ }^{8}$ African-American race was also associated with higher mortality rates. The finding of improved survival after ischaemic stroke and less severe stroke has also been noted in other studies. ${ }^{910}$

There are few reliable studies from sub-Saharan Africa (SSA). Two recent hospital-based studies of 30-day post-stroke case fatality, one from the Democratic Republic of Congo and one from Nigeria, found initial stroke severity, stroke sub-type and the presence of complications to be the strongest correlates of 30-day mortality. ${ }^{11,12}$ 
We are not aware of any previous studies of the correlates of case fatality from incident stroke cases in SSA. This study aimed to establish which factors were most strongly associated with poststroke case fatality. Hopefully, this information may help clinicians working in SSA to identify those at risk and allocate scarce resources more effectively.

\section{Participants and methods}

Participants were part of a stroke incidence study, details of which have been described previously. ${ }^{13}$ Ethical permission for the study was obtained from the National Institute of Medical Research in Tanzania and from the Newcastle and North Tyneside Joint Ethics Committee, UK.

\section{Recruitment}

The Tanzanian Stroke Incidence Project (TSIP) recruited cases from 15 June 2003 until 15 June 2006 and was conducted in two demographic surveillance sites (DSS): 52 villages in the Hai district of northern Tanzania; and 8 geographical divisions of the city of Dar-es-Salaam. ${ }^{13}$ The study described here is based on data from the Hai DSS, which has been described previously as part of the Adult Morbidity and Mortality Project (AMMP). ${ }^{14}$

From a population of 159 814, a total of 453 strokes were recorded in Hai during this period. Of these patients, 132 survived long enough post-stroke to be identified by TSIP. The remaining 321 were identified by a system of verbal autopsy, used to establish cause of death. The age-adjusted incidence was $108.6 / 100000$ (95\% CI 89.0 - 130.9). We used the standard WHO definition of stroke and included first-ever-in-a-lifetime and recurrent strokes. Only those who met the WHO criteria for stroke were included. ${ }^{15}$ Every effort was made to ensure case ascertainment was as complete as possible. ${ }^{13}$ Clearly only those cases identified by TSIP could be followed up, and so it those on which the results presented here are based.

Methods of long-term follow-up of stroke cases have been described previously. ${ }^{7}$ Case fatality rates at 28 days and 3 years were estimated to be $26.9 \%$ (95\% CI 19.3 - 34.5) and $75.4 \%$ (95\% CI 67.9 - 82.8) respectively.

\section{Measurement}

Patients were interviewed, assessed and examined as soon after the stroke event as possible. Frequently, however, this was a number of days post-stroke. A few cases were not identified as having had a stroke until visited by a healthcare worker or key informant some months later. Nevertheless, only 11 cases were interviewed at longer than 6 months post-stroke; the median time from incident stroke to assessment interview was 10 days (range 0 - 252 days).

Demographic information, social history, past medical history and information about events around the time of the stroke were recorded. This included time and date of stroke, duration and progression of onset, any epileptic seizure, loss of consciousness or headache, the nature of any help sought and details of any hospital admission. Accurate ascertainment of a person's age can be difficult in SSA. Where there was doubt, a series of prompts for memorable events was used to establish a year of birth and age calculated.

All subjects who were identified and interviewed prior to death underwent a medical assessment and examination that involved recording blood pressure, pulse rate and rhythm, cardiac auscultatory findings, height and weight, physical function (Barthel index ${ }^{16}$ ), neurological status (communication, swallowing, vision, muscle activity, sensation), electrocardiogram
(ECG), echocardiogram, chest X-ray and computed tomography (CT) scan.

For purposes of analysis Barthel index scores were divided into: 19 - 20 no disability; 15 - 18 moderate disability; and 0 - 14 severe disability. CT scans were analysed independently by a general radiologist at Kilimanjaro Christian Medical Centre, Tanzania (AJ) and a neuroradiologist from Newcastle General Hospital, UK and the diagnosis of cerebral infarct or haemorrhage compared. ${ }^{17}$ If opinions on the diagnosis differed, a consensus was reached. Intra-cerebral bleeds were classified as haemorrhagic strokes. Findings of ischaemia, haemorrhagic infarct, or no evidence of stroke were classified as infarction.

\section{Statistics}

The data were quantitative in nature and collected at a nominal, ordinal and interval/ratio level. They were analysed using standard statistical software, PASW Statistics 18 for Windows (SPSS, Chicago, IL, USA). With the exception of age and blood pressure, all variables were found to be non-normally distributed (Kolmogorov-Smirnov test) and so did not meet parametric assumptions.

The degree of correlation with case fatality was assessed using $\chi$-square test for dichotomous variables and point biserial correlation for all other variables. Data analysis was restricted to a bivariate level. Multivariate analysis, using logistic regression, failed to yield any further insight and a robust model could not be obtained. This is probably due in part to the relatively small number of cases.

\section{Results}

Of 64 subjects who had had a CT head scan within 15 days of incident stroke, 52 had had a cerebral infarct, 11 haemorrhage and 1 subarachnoid haemorrhage. The subject with a subarachnoid haemorrhage died after 10 days, and was excluded from further analysis due to the different aetiology of this stroke sub-type.

By 15 June 2009 ( 3 years after the end of the study), 87 cases from the remaining 131 (66.4\%, 95\% CI 58.3 - 74.5) had died. For 79, cause of death was recorded as stroke, including all subjects who died within the first 4 weeks post-stroke. One patient, whose date of death was unknown, was excluded from further analysis, leaving 130 remaining cases. For those for whom date of death was known $(n=86)$, the median time to death was 101 days (range 0 - 1765 ). Details of case fatality rates for the remaining 130 cases have been presented previously. ${ }^{7}$ The demographic and clinical characteristics of the entire cohort are shown in Table 1.

The study aimed to capture all persons who had a stroke within the study period; of these, 20 subjects were identified shortly after death had occurred and a further 2 died before a full interview could take place. A full assessment and examination were carried out on the remaining 109 cases. Case fatality at 28 days and 3 years was significantly correlated with a number of variables (see Tables 2 and 3 respectively).

As a consequence of death occurring prior to interview, certain data were available on only 15 of the 31(48.4\%) who died within 28 days of incident stroke. Data were available on 94 of the 99 (94.9\%) subjects who survived beyond 28 days. At 3 years a full data set was available for all 52 subjects still alive, but for only 57 of $78(73.1 \%)$ who had died. No further analysis of those with and without a pre-stroke history of diabetes, claudication, myocardial infarction, angina and asthma was undertaken. Although this was partly due to the small number of positive cases, it was also because in many populations in SSA such diagnoses are rare. Note that in our experience this scarcity reflects the limited ability of primary healthcare services to provide such a diagnosis, rather than the true prevalence of these conditions.

As previously reported, there was a difference in case fatality between stroke sub-types at 28 days, with 3 deaths after haemorrhagic 
Table 1. Characteristics of the study population $(N=130)$

\begin{tabular}{|c|c|}
\hline \multicolumn{2}{|l|}{ Demographics } \\
\hline Sex & $69(53.1 \%)$ male, $61(46.9 \%)$ female \\
\hline Age (years) & Mean 68.8 , range $23-100, S D \pm 14.80$ \\
\hline Ethnic group & 109 (83.8\%) Chagga, 11 Zaramo, 4 Pare, 2 Masai, 1 Somali, 1 Sambaa, 1 Mgogo, 1 Nyiramba \\
\hline Religion & 115 (88.5\%) Christian, 14 Muslim, 1 Hindu \\
\hline \multicolumn{2}{|l|}{ Previous medical history } \\
\hline Barthel index prior to stroke $(n=95)$ & $84(88.4 \%)$ no disability, $3(3.2 \%)$ moderate disability, 6 (6.3\%) severe disability \\
\hline Previous stroke $(n=130)$ & $16(12.3 \%)$ one previous stroke, $3(2.3 \%)$ two previous strokes \\
\hline History of hypertension $(n=93)$ & $28(30.1 \%)$ yes, 65 no \\
\hline $\begin{array}{l}\text { Blood pressure measured in } 12 \text { months } \\
\text { before stroke }(n=106)\end{array}$ & $59(55.7 \%)$ yes, 47 no \\
\hline $\begin{array}{l}\text { Receiving hypertensives prior to stroke } \\
(n=108)\end{array}$ & $40(37.0 \%)$ yes, 68 no \\
\hline Angina $(n=109)$ & 109 no \\
\hline Myocardial infarction $(\mathrm{n}=109)$ & 109 no \\
\hline Claudication $(n=109)$ & 109 no \\
\hline Asthma $(n=109)$ & $3(2.8 \%)$ yes, 106 no \\
\hline Diabetes $(n=109)$ & $8(7.3 \%)$ yes, 101 no \\
\hline \multicolumn{2}{|l|}{ Social history } \\
\hline Smoking $(n=109)$ & $\begin{array}{l}24(22.0 \%) \text { current smoker, } 7(6.4 \%) \text { stopped in last } 12 \text { months, } 78 \text { (71.6\%) never smoked/stopped } \\
\text { over } 12 \text { months ago }\end{array}$ \\
\hline Alcohol intake $(n=104)$ & $\begin{array}{l}44(42.3 \%) \text { non-drinker, } 18(17.3 \%) \text { only weekends and holidays, } 21(20.2 \%) \text { less than } 4 \\
\text { times per week, } 21(20.2 \%) \text { more than } 4 \text { times per week }\end{array}$ \\
\hline \multicolumn{2}{|l|}{ Current level of functioning (at interview) } \\
\hline Barthel index ${ }^{*}(n=100)$ & $16(16.0 \%)$ no disability, $9(9.0 \%)$ moderate disability, 75 (75.0\%) severe disability \\
\hline Systolic blood pressure $(n=104)$ & Mean 153.6, range $60-284, \mathrm{SD} \pm 36.50$ \\
\hline Diastolic blood pressure $(n=104)$ & Mean 95.1 , range $50-180, \mathrm{SD} \pm 23.60$ \\
\hline Expressive dysphasia $(n=109)$ & $56(51.4 \%)$ none, $13(11.9 \%)$ minor problems, $40(36.7 \%)$ major problems/unable to assess \\
\hline Receptive dysphasia $(n=109)$ & $72(66.1 \%)$ none, $11(10.1 \%)$ minor problems, $26(23.9 \%)$ major problems/unable to assess \\
\hline Dysphagia $(n=109)$ & $86(78.9 \%)$ normal, $4(3.7 \%)$ abnormal, $2(1.8 \%)$ impossible, $17(15.6 \%)$ unable to assess \\
\hline Dysarthria $(n=109)$ & $\begin{array}{l}45(41.3 \%) \text { none, } 23(21.1 \%) \text { slight slurring, } 4(3.7 \%) \text { difficult to understand, } 11(10.1 \%) \\
\text { incomprehensible, } 26(23.9 \%) \text { aphasic/unable to assess }\end{array}$ \\
\hline Gaze paresis $(n=107)$ & $2(1.9 \%)$ yes, $90(84.1 \%)$ no, $15(14.0 \%)$ unable to assess \\
\hline Fits post-stroke $(n=109)$ & $1(0.9 \%)$ yes, 108 no \\
\hline Hearing $(n=93)$ & $87(93.5 \%)$ normal, 6 (6.5\%) slightly impaired \\
\hline Mobility $(n=107)$ & $\begin{array}{l}50(46.7 \%) \text { bed bound, } 17(15.9 \%) \text { chair bound, } 10 \text { (9.3\%) house bound, } 17 \text { (15.9\%) limited } \\
\text { mobility, } 13(12.1 \%) \text { independently mobile }\end{array}$ \\
\hline Confusion $(n=107)$ & $65(60.7 \%)$ not confused, $12(11.2 \%)$ minor problems, $30(28.0 \%)$ major problems/unable to assess \\
\hline Lift upper limb against gravity $(n=108)$ & $42(38.9 \%)$ can lift both limbs, $43(39.8 \%)$ cannot lift affected limb, 23 (21.3\%) can lift neither limb \\
\hline Lift lower limbs against gravity $(n=108)$ & $47(43.5 \%)$ can lift neither limb, $38(35.2 \%)$ cannot lift affected limb, $23(21.3 \%)$ can lift both limbs \\
\hline
\end{tabular}

stroke $(27.3 \%)$ and 10 subsequent to cerebral infarct (19.2\%). Threeyear case fatalities were 4 (36.4\%) for haemorrhage and $26(50.0 \%)$ for infarct $\left(\chi^{2}=0.677, p=0.515\right) .^{7}$ Neither of these results reached statistical significance, though this is likely due in part to the small numbers involved (Type II error). Of 93 subjects who underwent ECG, only 4 had atrial fibrillation (AF). Due to the small number of subjects, no further analysis of those with and without AF was undertaken.

\section{Discussion}

This is the first study of the correlates of post-stroke case fatality in an incident cohort of stroke cases in SSA. The results of this study 
Table 2. Twenty-eight-day case fatality

\begin{tabular}{|c|c|c|c|}
\hline & Case fatality & Not case fatality & Significance \\
\hline $\begin{array}{l}\text { Sex } \\
\qquad(n=130)\end{array}$ & 15 male, 16 female & 54 male, 45 female & $\begin{array}{l}\chi^{2}(1)=0.299 \\
p=0.682\end{array}$ \\
\hline $\begin{array}{l}\text { Age } \\
\qquad(n=130)\end{array}$ & Mean $70.5(95 \%$ CI $65.8-75.2)$ & Mean $68.2(95 \%$ CI $65.1-71.2)$ & $\begin{array}{l}r=0.066 \\
p=0.456\end{array}$ \\
\hline $\begin{array}{l}\text { Smoking } \\
\qquad(n=109,15 \text { dead, } 94 \text { alive })\end{array}$ & $\begin{array}{l}8 \text { current smoker, } 1 \text { stopped in last } \\
12 \text { months, } 6 \text { never smoked/stopped } \\
\text { over } 12 \text { months ago }\end{array}$ & $\begin{array}{l}16 \text { current smoker, } 6 \text { stopped in last } \\
12 \text { months, } 72 \text { never smoked/stopped } \\
\text { over } 12 \text { months ago }\end{array}$ & $\begin{array}{l}r=0.302 \\
p=0.001\end{array}$ \\
\hline $\begin{array}{l}\text { Alcohol intake } \\
\qquad(n=103,15 \text { dead, } 88 \text { alive })\end{array}$ & $\begin{array}{l}6 \text { non-drinker, } 1 \text { only weekends and } \\
\text { holidays, } 4 \text { less than } 4 \text { times per week, } \\
4 \text { more than } 4 \text { times per week }\end{array}$ & $\begin{array}{l}38 \text { non-drinker, } 17 \text { only weekends } \\
\text { and holidays, } 17 \text { less than } 4 \text { times per } \\
\text { week, } 16 \text { more than } 4 \text { times per week }\end{array}$ & $\begin{array}{l}r=0.083 \\
p=0.407\end{array}$ \\
\hline $\begin{array}{l}\text { Barthel index prior to stroke } \\
\qquad(n=92,12 \text { dead, } 80 \text { alive })\end{array}$ & $\begin{array}{l}10 \text { no disability, } 1 \text { moderate disability, } \\
1 \text { severe disability }\end{array}$ & $\begin{array}{l}73 \text { no disability, } 2 \text { moderate disability, } \\
5 \text { severe disability }\end{array}$ & $\begin{array}{l}r=-0.065 \\
p=0.537\end{array}$ \\
\hline $\begin{array}{l}\text { Barthel index at interview } \\
\qquad(n=100,12 \text { dead, } 88 \text { alive })\end{array}$ & 1 no disability, 11 severe disability & $\begin{array}{l}15 \text { no disability, } 9 \text { moderate disability, } \\
64 \text { severe disability }\end{array}$ & $\begin{array}{l}r=-0.277 \\
p=0.005\end{array}$ \\
\hline $\begin{array}{l}\text { Systolic blood pressure } \\
\qquad(n=104,15 \text { dead, } 89 \text { alive })\end{array}$ & $\begin{array}{l}\text { Mean } 155.47, \mathrm{SD} \pm 23.308 \text {, } \\
\text { range } 100-195\end{array}$ & $\begin{array}{l}\text { Mean } 153.29, \mathrm{SD} \pm 38.351 \text {, } \\
\text { range } 80-260\end{array}$ & $\begin{array}{l}r=0.021 \\
p=0.832\end{array}$ \\
\hline $\begin{array}{l}\text { Diastolic blood pressure } \\
\qquad(n=104,15 \text { dead, } 89 \text { alive })\end{array}$ & $\begin{array}{l}\text { Mean } 96.13, \mathrm{SD} \pm 28.206 \text {, } \\
\text { range } 60-181\end{array}$ & $\begin{array}{l}\text { Mean } 95.00, \mathrm{SD} \pm 22.928 \\
\text { range } 50-181\end{array}$ & $\begin{array}{l}r=0.017 \\
p=0.864\end{array}$ \\
\hline $\begin{array}{l}\text { Expressive dysphasia } \\
\qquad(n=109,15 \text { dead, } 94 \text { alive })\end{array}$ & $\begin{array}{l}3 \text { none, } 12 \text { major problems/unable } \\
\text { to assess }\end{array}$ & $\begin{array}{l}53 \text { none, } 13 \text { minor problems, } 28 \text { major } \\
\text { problems/unable to assess }\end{array}$ & $\begin{array}{l}r=0.370 \\
p<0.001\end{array}$ \\
\hline $\begin{array}{l}\text { Receptive dysphasia } \\
\qquad(n=109,15 \text { dead, } 94 \text { alive })\end{array}$ & $\begin{array}{l}4 \text { none, } 1 \text { minor problems, } 10 \text { major } \\
\text { problems/unable to assess }\end{array}$ & $\begin{array}{l}68 \text { none, } 10 \text { minor problems, } 16 \text { major } \\
\text { problems/unable to assess }\end{array}$ & $\begin{array}{l}r=0.408 \\
p<0.001\end{array}$ \\
\hline $\begin{array}{l}\text { Dysphagia } \\
\qquad(n=109,15 \text { dead, } 94 \text { alive })\end{array}$ & $\begin{array}{l}5 \text { normal, } 1 \text { abnormal, } 2 \text { impossible, } \\
7 \text { unable to assess }\end{array}$ & $\begin{array}{l}81 \text { normal, } 3 \text { abnormal, } 10 \text { unable } \\
\text { to assess }\end{array}$ & $\begin{array}{l}r=0.408 \\
p<0.001\end{array}$ \\
\hline $\begin{array}{l}\text { Dysarthria } \\
\qquad(n=109,15 \text { dead, } 94 \text { alive })\end{array}$ & $\begin{array}{l}3 \text { none, } 1 \text { slight slurring, } \\
3 \text { incomprehensible, } 8 \text { aphasic/ } \\
\text { unable to assess }\end{array}$ & $\begin{array}{l}42 \text { none, } 22 \text { slight slurring, } 4 \text { difficult } \\
\text { to understand, } 8 \text { incomprehensible, } \\
18 \text { aphasic/unable to assess }\end{array}$ & $\begin{array}{l}r=0.306 \\
p=0.001\end{array}$ \\
\hline $\begin{array}{l}\text { Gaze paresis } \\
\qquad(n=108,15 \text { dead, } 93 \text { alive })\end{array}$ & 8 no, 7 unable to assess & 82 no, 2 yes, 9 unable to assess & $\begin{array}{l}r=0.365 \\
p<0.001\end{array}$ \\
\hline $\begin{array}{l}\text { Hearing } \\
\qquad(n=93,9 \text { dead, } 84 \text { alive })\end{array}$ & 7 normal, 2 slightly impaired & 80 normal, 4 slightly impaired & $\begin{array}{l}r=0.310 \\
p=0.043\end{array}$ \\
\hline $\begin{array}{l}\text { Mobility } \\
\qquad(n=107,14 \text { dead, } 93 \text { alive })\end{array}$ & 13 bed bound, 1 independently mobile & $\begin{array}{l}37 \text { bed-bound, } 17 \text { chair-bound, } \\
10 \text { house-bound, } 17 \text { limited mobility, } \\
12 \text { independently mobile }\end{array}$ & $\begin{array}{l}r=-0.288 \\
p=0.011\end{array}$ \\
\hline $\begin{array}{l}\text { Confusion } \\
\qquad(n=107,14 \text { dead, } 93 \text { alive })\end{array}$ & $\begin{array}{l}5 \text { not confused, } 9 \text { major problems/unable } \\
\text { to assess }\end{array}$ & $\begin{array}{l}60 \text { not confused, } 12 \text { minor problems, } \\
21 \text { major problems/unable to assess }\end{array}$ & $\begin{array}{l}r=0.243 \\
p=0.011\end{array}$ \\
\hline $\begin{array}{l}\text { Lift upper limb against gravity } \\
\qquad(n=108,15 \text { dead, } 93 \text { alive })\end{array}$ & $\begin{array}{l}2 \text { can lift both limbs, } 4 \text { cannot lift affected } \\
\text { limb, } 9 \text { can lift neither limb }\end{array}$ & $\begin{array}{l}40 \text { can lift both limbs, } 39 \text { cannot lift } \\
\text { affected limb, } 14 \text { can lift neither limb }\end{array}$ & $\begin{array}{l}r=0.342 \\
p<0.001\end{array}$ \\
\hline $\begin{array}{l}\text { Lift lower limbs against gravity } \\
\qquad(n=108,15 \text { dead, } 93 \text { alive })\end{array}$ & $\begin{array}{l}2 \text { can lift both limbs, } 4 \text { cannot lift affected } \\
\text { limb, } 9 \text { can lift neither limb }\end{array}$ & $\begin{array}{l}45 \text { can lift both limbs, } 34 \text { cannot lift } \\
\text { affected limb, } 14 \text { can lift neither limb }\end{array}$ & $\begin{array}{l}r=0.358 \\
p<0.001\end{array}$ \\
\hline
\end{tabular}

are more representative of the wider community of stroke cases than results from previous hospital-based studies. ${ }^{11,12}$

Among the 130 patients who survived long enough to be assessed, death was associated with a number of predictor variables. However, smoking history (28-day case fatality) and age at stroke (3-year case fatality) were the only pre-stroke markers for increased case fatality risk. The majority of correlates related to the severity of the stroke itself and its neurological sequelae and motor complications. These, in turn, are probably a consequence of stroke severity.

Our results support findings from studies in high-income countries. ${ }^{9,10,18-21}$ Speech, language and swallowing problems, reduced muscle power, and reduced functional ability (Barthel index) at post-stroke assessment were all significantly associated with death. Motor impairments are likely to lead to long-term immobility, poor nutrition, and reduced social interaction. The resulting co-morbidity probably contributes to higher case fatality rates and we hypothesise that death could be reduced by improved active rehabilitation following stroke. ${ }^{3}$ Indeed, studies of stroke survivors in the UK, Japan and Hungary have noted the link between survival and active poststroke medical management. .1,22 $^{2}$

While other authors have noted most of these variables as predictive of post-stroke outcome, it is those factors which were 
Table 3. Three-year case fatality

\begin{tabular}{|c|c|c|c|}
\hline & Case fatality & Not case fatality & Significance \\
\hline $\begin{array}{l}\text { Sex } \\
\qquad(n=130)\end{array}$ & 42 male, 36 female & 27 male, 25 female & $\begin{array}{l}\chi^{2}(1)=0.107 \\
p=0.859\end{array}$ \\
\hline $\begin{array}{l}\text { Age } \\
\qquad(n=30)\end{array}$ & Mean $71.2(95 \%$ CI 68.0 - 74.3) & Mean $65.0(95 \%$ CI 60.7 - 69.4) & $\begin{array}{l}r=0.198 \\
p=0.024\end{array}$ \\
\hline $\begin{array}{l}\text { Smoking } \\
\qquad(n=109,57 \text { dead, } 52 \text { alive })\end{array}$ & $\begin{array}{l}16 \text { current smoker, } 2 \text { stopped in last } \\
12 \text { months, } 39 \text { never smoked/stopped } \\
\text { over } 12 \text { months ago }\end{array}$ & $\begin{array}{l}9 \text { current smoker, } 5 \text { stopped in last } \\
12 \text { months, } 38 \text { never smoked/stopped } \\
\text { over } 12 \text { months ago }\end{array}$ & $\begin{array}{l}r=0.083 \\
p=0.393\end{array}$ \\
\hline $\begin{array}{l}\text { Alcohol intake } \\
\qquad(n=103,51 \text { dead, } 52 \text { alive })\end{array}$ & $\begin{array}{l}25 \text { non-drinker, } 5 \text { only weekends and } \\
\text { holidays, } 10 \text { less than } 4 \text { times per week, } \\
11 \text { more than } 4 \text { times per week }\end{array}$ & $\begin{array}{l}19 \text { non-drinker, } 13 \text { only weekends } \\
\text { and holidays, } 10 \text { less than } 4 \text { times per } \\
\text { week, } 10 \text { more than } 4 \text { times per week }\end{array}$ & $\begin{array}{l}r=0.035 \\
p=0.729\end{array}$ \\
\hline $\begin{array}{l}\text { Barthel index prior to stroke } \\
\qquad(n=92,47 \text { dead, } 45 \text { alive })\end{array}$ & $\begin{array}{l}40 \text { no disability, } 2 \text { moderate disability, } \\
5 \text { severe disability }\end{array}$ & $\begin{array}{l}43 \text { no disability, } 1 \text { moderate disability, } \\
1 \text { severe disability }\end{array}$ & $\begin{array}{l}r=-0.183 \\
p=0.082\end{array}$ \\
\hline $\begin{array}{l}\text { Barthel index at interview } \\
\qquad(n=100,50 \text { dead, } 50 \text { alive })\end{array}$ & $\begin{array}{l}5 \text { no disability, } 3 \text { moderate disability, } \\
42 \text { severe disability }\end{array}$ & $\begin{array}{l}11 \text { no disability, } 6 \text { moderate disability, } \\
33 \text { severe disability }\end{array}$ & $\begin{array}{l}r=-0.330 \\
p=0.001\end{array}$ \\
\hline $\begin{array}{l}\text { Systolic blood pressure } \\
\qquad(n=104,54 \text { dead, } 50 \text { alive })\end{array}$ & $\begin{array}{l}\text { Mean } 158.82, \mathrm{SD} \pm 39.370 \\
\text { range } 80-260\end{array}$ & $\begin{array}{l}\text { Mean } 147.86, \mathrm{SD} \pm 32.494 \text {, } \\
\text { range } 80-220\end{array}$ & $\begin{array}{l}r=0.151 \\
p=0.125\end{array}$ \\
\hline $\begin{array}{l}\text { Diastolic blood pressure } \\
\qquad(n=104,54 \text { dead, } 50 \text { alive })\end{array}$ & $\begin{array}{l}\text { Mean } 95.76, \pm \text { SD } 25.171 \text {, } \\
\text { range } 50-181\end{array}$ & $\begin{array}{l}\text { Mean } 94.50, \mathrm{SD} \pm 21.986 \text {, } \\
\text { range } 50-181\end{array}$ & $\begin{array}{l}r=0.027 \\
p=0.786\end{array}$ \\
\hline $\begin{array}{l}\text { Expressive dysphasia } \\
\qquad(n=109,57 \text { dead, } 52 \text { alive })\end{array}$ & $\begin{array}{l}23 \text { none, } 6 \text { minor problems, } 28 \text { major } \\
\text { problems/unable to assess }\end{array}$ & $\begin{array}{l}33 \text { none, } 7 \text { minor problems, } 12 \text { major } \\
\text { problems/unable to assess }\end{array}$ & $\begin{array}{l}r=0.256 \\
p=0.007\end{array}$ \\
\hline $\begin{array}{l}\text { Receptive dysphasia } \\
\qquad(n=109,57 \text { dead, } 52 \text { alive })\end{array}$ & $\begin{array}{l}29 \text { none, } 6 \text { minor problems, } 22 \text { major } \\
\text { problems/unable to assess }\end{array}$ & $\begin{array}{l}43 \text { none, } 5 \text { minor problems, } 4 \text { major } \\
\text { problems/unable to assess }\end{array}$ & $\begin{array}{l}r=0.347 \\
p<0.001\end{array}$ \\
\hline $\begin{array}{l}\text { Dysphagia } \\
\qquad(n=109,57 \text { dead, } 52 \text { alive })\end{array}$ & $\begin{array}{l}36 \text { normal, } 5 \text { dysphagic, } 2 \text { aphagic, } \\
14 \text { unable to assess }\end{array}$ & 50 normal, 2 unable to assess & $\begin{array}{l}r=0.347 \\
p<0.001\end{array}$ \\
\hline $\begin{array}{l}\text { Dysarthria } \\
\qquad(n=109,57 \text { dead, } 52 \text { alive })\end{array}$ & $\begin{array}{l}18 \text { none, } 10 \text { slight slurring, } 2 \text { difficult } \\
\text { to understand, } 10 \text { incomprehensible, } \\
17 \text { aphasic/unable to assess }\end{array}$ & $\begin{array}{l}27 \text { none, } 13 \text { slight slurring, } 2 \text { difficult } \\
\text { to understand, } 1 \text { incomprehensible, } \\
9 \text { aphasic/unable to assess }\end{array}$ & $\begin{array}{l}r=0.242 \\
p=0.011\end{array}$ \\
\hline $\begin{array}{l}\text { Gaze paresis } \\
\qquad(n=108,56 \text { dead, } 52 \text { alive })\end{array}$ & 42 no, 1 yes, 13 unable to assess & 48 no, 1 yes, 3 unable to assess & $\begin{array}{l}r=0.204 \\
p=0.034\end{array}$ \\
\hline $\begin{array}{l}\text { Hearing } \\
\qquad(n=93,44 \text { dead, } 49 \text { alive })\end{array}$ & 40 normal, 4 slightly impaired & 47 normal, 2 slightly impaired & $\begin{array}{l}r=0.102 \\
p=0.332\end{array}$ \\
\hline $\begin{array}{l}\text { Mobility } \\
\qquad(n=107,55 \text { dead, } 52 \text { alive })\end{array}$ & $\begin{array}{l}34 \text { bed-bound, } 9 \text { chair-bound, } \\
2 \text { house-bound, } 6 \text { limited } \\
\text { mobility, } 4 \text { independently mobile }\end{array}$ & $\begin{array}{l}17 \text { bed bound, } 8 \text { chair bound, } \\
7 \text { house bound, } 11 \text { limited } \\
\text { mobility, } 9 \text { independently mobile }\end{array}$ & $\begin{array}{l}r=-0.328 \\
p=0.001\end{array}$ \\
\hline $\begin{array}{l}\text { Confusion } \\
\qquad(n=107,55 \text { dead, } 52 \text { alive })\end{array}$ & $\begin{array}{l}29 \text { not confused, } 4 \text { minor problems, } \\
22 \text { major problems/unable to assess }\end{array}$ & $\begin{array}{l}36 \text { not confused, } 8 \text { minor problems, } \\
8 \text { major problems/unable to assess }\end{array}$ & $\begin{array}{l}r=0.224 \\
p=0.020\end{array}$ \\
\hline $\begin{array}{l}\text { Lift upper limb against gravity } \\
\qquad(n=108,56 \text { dead, } 52 \text { alive })\end{array}$ & $\begin{array}{l}16 \text { can lift both limbs, } 22 \text { cannot lift } \\
\text { affected limb, } 18 \text { can lift neither limb }\end{array}$ & $\begin{array}{l}26 \text { can lift both limbs, } 21 \text { cannot lift } \\
\text { affected limb, } 5 \text { can lift neither limb }\end{array}$ & $\begin{array}{l}r=0.291 \\
p=0.002\end{array}$ \\
\hline $\begin{array}{l}\text { Lift lower limbs against gravity } \\
\qquad(n=108,56 \text { dead, } 52 \text { alive })\end{array}$ & $\begin{array}{l}29 \text { can lift both limbs, } 18 \text { cannot lift } \\
\text { affected limb, } 5 \text { can lift neither limb }\end{array}$ & $\begin{array}{l}18 \text { can lift both limbs, } 20 \text { cannot lift } \\
\text { affected limb, } 18 \text { can lift neither limb }\end{array}$ & $\begin{array}{l}r=0.298 \\
p=0.002\end{array}$ \\
\hline
\end{tabular}

not associated with case fatality which are perhaps the most interesting. ${ }^{10,23-25}$ Most reports of post-stroke case fatality from high-income countries suggest that age is a significant predictor of short-term case fatality. 19,20,23,26,27 Within the TSIP cohort, 28-day case fatality was not linked to gender or age, suggesting that the sequelae of the stroke itself override normal gender- and age-related differences in life expectancy. At 3 years post-stroke, gender was not associated with case fatality, though age was.

We were able to collect information on pre-stroke levels of disability and function (Barthel index) in almost three-quarters of cases. There was no association between pre-stroke disability and case fatality at either 28 days or 3 years, which is in contrast to the findings of Longstreth et al., ${ }^{8}$ who found pre-stroke walking ability to be a marker for death at 7 years follow-up. The influence of the stroke itself on case fatality rates appears to override levels of prestroke function in our cohort. It is possible that better immediate post-stroke care may help to improve survival rates.

At 28 days post-stroke, those who had suffered haemorrhagic stroke had a greater case fatality rate than those who had suffered a stroke as a result of a cerebral infarct. These findings support previous 
hospital-based studies in SSA. ${ }^{24}$ They are also broadly similar to those reported by Feigin et al. ${ }^{5}$ in a systematic review of population-based incidence studies. In the period 2000 - 2008, mean case fatality from ischaemic stroke, primary intracerebral haemorrhage and subarachnoid haemorrhage was respectively $14.3 \%, 41.0 \%$ and $30.0 \%$ in high-income countries, and $16.7 \%, 38.7 \%$ and $43.9 \%$ in low- to middle-income countries.

We acknowledge that our findings are based on those patients who survived long enough to be identified, interviewed and assessed by TSIP, and that those identified only after death (by verbal autopsy) might have had a different stroke aetiology, and thus risk factor profile. Only by identifying a large community-based cohort, and following them up to see which individuals went on to have a stroke, could information on those who died rapidly following stroke be obtained. ${ }^{8,28}$

Nevertheless, we present the first large-scale study of risk factors for post-stroke case fatality from within an incident cohort in SSA, and we feel it adds substantially to current knowledge. We were unable to identify independent predictors of case fatality using logistic regression. A robust and reliable model could not be constructed, in part due to the small number of cases.

The study described here was part of a stroke incidence study. It was designed to collect information once a stroke had occurred. More objective information on pre-stroke levels of functioning and morbidity would have added substantially to our results. However, in SSA, detailed medical records are not generally available and retrospective medical data are often unreliable due to poor coverage of healthcare services. Such data would only be collected from a large-scale prospective longitudinal cohort study of disease risk factors.

In conclusion, correlates of 28-day and 3-year post-stroke case fatality were similar encompassing pre-stroke smoking and the neurological deficit caused by the stroke. In contrast with reports from high-income countries, pre-stroke demographics and levels of function were not significantly associated with case fatality. The main reason for this contrast seems likely to reside within the lower levels of immediate post-stroke care available to our cohort, which may reflect the situation in SSA as a whole.

Acknowledgements. We wish to acknowledge all patients, relatives and carers who participated in this study.

Funding. This work was funded by a grant from the Wellcome Trust (grant number 066939).

\section{References}

1. Carandang R, Seshadri S, Beiser A, et al. Trends in incidence, lifetime risk, severity, and 30-day mortality of stroke over the past 50 years. JAMA 2006;296(24):2939-2946. [http://dx.doi.org/10.1001/ jama.296.24.2939]

2. Rothwell PM, Coull AJ, Giles MF, et al. Change in stroke incidence, mortality, case fatality, severity, and risk factors in Oxfordshire, UK from 1981 to 2004 (Oxford Vascular Study). Lancet 2004;363(9425):19251933. [http://dx.doi.org/10.1016/S0140-6736(04)16405-2]

3. Hallström B, Jönsson AC, Nerbrand C, Norrving B, Lindgren A. Stroke incidence and survival in the beginning of the 21 st century in southern Sweden: comparisons with the late 20 th century and projection into the future. Stroke 2008;39(1):10-15. [http://dx.doi.org/10.1161/STROKEAHA.107.491779]

4. Johnston SC, Mendis S, Mathers CD. Global variation in stroke burden and mortality: estimates from monitoring, surveillance, and modelling. Lancet Neurol 2009;8(4):345-354. [http://dx.doi.org/10.1016 S1474-4422(09)70023-7]

5. Feigin VL, Lawes CM, Bennett DA, Barker-Collo SL, Parag V. Worldwide stroke incidence and early case fatality reported in 56 population-based studies: a systematic review. Lancet Neurol 2009;8(4):355-369. [http://dx.doi.org/10.1016/S1474-4422(09)70025-0]

6. Connor MD, Walker R, Modi G, Warlow CP. Burden of stroke in black populations in sub-Saharan Africa. Lancet Neurol 2007;6(3):269-278. [http://dx.doi.org/10.1016/S1474-4422(07)70002-9]

7. Walker R, Jusabani A, Aris E, et al. Post-stroke case fatality within an incident population in rural Tanzania. J Neurol Neurosurgery Psychiatry 2011;82(9):1001-1005. [http://dx.doi.org/10.1136/jnnp.2010.231944]

8. Longstreth Wt, Jr., Bernick C, Fitzpatrick A, et al. Frequency and predictors of stroke death in 5,888 participants in the Cardiovascular Health Study. Neurology 2001;56(3):368-375.

9. Kolominsky-Rabas PL, Weber M, Gefeller O, Neundoerfer B, Heuschmann PU. Epidemiology of ischemic stroke subtypes according to TOAST criteria: incidence, recurrence, and long-term survival in ischemic stroke subtypes: a population-based study. Stroke 2001;32(12):2735-2740.

10. Appelros P, Nydevik I, Viitanen M. Poor outcome after first-ever stroke: predictors for death, dependency, and recurrent stroke within the first year. Stroke 2003;34(1):122-126.

11. Longo-Mbenza B, Lelo Tshinkwela M, Mbuilu Pukuta J. Rates and predictors of stroke-associated case Longo-Mbenza B, Lelo Tshinkwela M, Mbuilu Pukuta J. Rates and predicto
fatality in black Central African patients. Cardiovasc J Afr 2008;19(2):72-76.

12. Wahab KW, Okubadejo NU, Ojini FI, Danesi MA. Predictors of short-term intra-hospital case fatality Wahab KW, Okubadejo NU, Ojini FI, Danesi MA. Predictors of short-term intra-hospital case fatality
following first-ever acute ischaemic stroke in Nigerians. J Coll Physicians Surg Pak 2008;18(12):755-758. following first-ever acute ischaemic stroke

13. Walker $\mathrm{R}$, Whiting $\mathrm{D}$, Unwin $\mathrm{N}$, et al. Stroke incidence in rural and urban Tanzania: a prospective Walker R, Whiting D, Unwin N, et al. Stroke incidence in rural and urban Tanzania: a prospective,
community-based study. Lancet Neurol 2010;9(8):786-792. [http://dx.doi.org/10.1016/S1474community-based

14. Adult Morbidity and Mortality Project (AMMP). Policy Implications of Adult Morbidity and Mortality: Final Report. Dar-es-Salaam: Tanzanian Ministry of Health; 2004 [updated 3rd April 2010]. http:// Final Report. Dar-es-Salaam:

15. World Health Organization. International Statistical Classification of Disease and Related Health Problems, 10th Revision, Volume 2. Geneva, Switzerland: World Health Organization, 1993.

Problems, 10th Revision, Volume 2. Geneva, Switzerland: World Health Organization, 1993.
6. Mahoney FI, Barthel D. Functional evaluation: The Barthel Index. Maryland State Med J 1965;14:56-61.

16. Mahoney FI, Barthel D. Functional evaluation: The Barthel Index. Maryland State Med J 1965; 14:56-61.
17. Walker RW, Jusabani A, Aris E, Gray WK, Mitra D, Swai M. A prospective study of stroke sub-type from . Walker RW, Jusabani A, Aris E, Gray WK, Mitra D, Swai M. A prospective study of
within an incident population in Tanzania. S Afr Med J 2011;101(5):338-344.

within an incident population in Tanzania. S Afr Med J 2011;101(5):338-344.
18. Smithard DG, Smeeton NC, Wolfe CD. Long-term outcome after stroke: does dysphagia matter? Age Smithard DG, Smeeton NC, Wolfe CD. Long-term outcome after stroke: does
Ageing 2007;36(1):90-94. [http://dx.doi.org/10.1093/ageing/afl $49</$ ArticleId]

Ageing 2007;36(1):90-94. [http://dx.doi.org/10.1093/ageing/afl149</ArticleId] 9. Nedeltchev K, Renz N, Karameshev A, et al. Predictors of early mortality after acute ischaemic stroke. Swiss Med Wkly 2010;140(17-18):254-259. [http://dx.doi.org/smw-12919]

20. De Jong G, Van Raak L, Kessels F, Lodder J. Stroke subtype and mortality. A follow-up study in 998 patients with a first cerebral infarct. J Clin Epidemiol 2003;56(3):262-268.

21. Sarker SJ, Heuschmann PU, Burger I, et al. Predictors of survival after haemorrhagic stroke in multi-ethnic population: the South London Stroke Register (SLSR). J Neurol Neurosurg Psychiatry 2008; $79(3): 260-265$

22. Ováry C, Suzuki K, Nagy Z. Regional differences in incidence rates, outcome predictors and survival of stroke. Neuroepidemiology 2004;23(5):240-246. [http://dx.doi.org/10.1159/000079950]

23. Kelly-Hayes M, Wolf PA, Kannel WB, Sytkowski P, Dagostino RB, Gresham GE. Factors influencing survival and need for institutionalization following stroke: the Framingham Study. Arch Phys Med Rehabil 1988;69(6):415-418

24. Owolabi MO, Ugoya S, Platz T. Racial disparity in stroke risk factors: the Berlin-Ibadan experience; a retrospective study. Acta Neurol Scand 2009;119(2):81-87. [http://dx.doi.org/10.1111/j.16000404.2008.01077.x]

25. Walker RW, Rolfe M, Kelly PJ, George MO, James OF. Mortality and recovery after stroke in the Gambia. Stroke 2003;34(7):1604-1609. [http://dx.doi.org/10.1161/01.STR.0000077943.63718.67]

26. Collins TC, Petersen NJ, Menke TJ, Souchek J, Foster W, Ashton CM. Short-term, intermediate-term, and long-term mortality in patients hospitalized for stroke. J Clin Epidemiol 2003;56:81-87.

27. Zuliani G, Cherubini A, Ranzini M, Ruggiero C, Atti AR, Fellin R. Risk factors for short-term mortality in older subjects with acute ischemic stroke. Gerontology 2006;52(4):231-236. [http://dx.doi. org $/ 10.1159 / 000093655$

28. Sacco RL, Wolf PA, Kannel WB, McNamara PM. Survival and recurrence following stroke. The Framingham study. Stroke 1982;13(3):290-295

Accepted 11 October 2012. 